\title{
Spinal cord re-treatments using photon and proton based radiotherapy: LQ-derived tolerance doses.
}

Jones $\mathrm{B}^{1,2}$ and Hopewell $\mathrm{JW}^{2}$

1. CRUK-MRC Oxford Centre, Gray Laboratory, Department of Oncology, University of Oxford, Old Road Research Campus Research Building, Roosevelt Drive, Oxford OX3 7DQ

2. Green Templeton College, University of Oxford, 43 Woodstock Road, Oxford, OX2 6HG.

Key words: radiotherapy, radiobiology, spinal cord, proton therapy.

Correspondence to Professor Bleddyn Jones at above address:

Bleddyn.Jones@oncology.ox.ac.uk 


\begin{abstract}
Re-treatment, using megavoltage photon radiotherapy, can benefit carefully selected patients with new or recurrent tumours. Such re-treatments may involve the further exposure of tissues such as the brain or spinal cord.
\end{abstract}

A time-dependent model has been developed, which incorporates data from all published radiobiological experiments concerned with the in vivo re-irradiation of the spinal cord using photons. It allows an estimation of the increasing recovery in tissue tolerance with elapsed time after the initial treatment course. In accordance with the experimental evidence, the recovery rate depends on the biological effective dose (BED) of the initial treatment. Various degrees of conservatism have been introduced in the model to allow for potential changes in CNS tissue tolerance due to patient age, chemotherapy, surgery etc.

An estimation of the re-treatment dose-fractionation schedule is made easier by the use of a downloadable Graphical User Interface (GUI). Worked examples of its use are given for conventional photon (x-ray) based treatments, and also for protons, where relative biological effectiveness (RBE) considerations must be respected within the BED estimates. The model provides boundary conditions for clinical practice. The responsible clinician can choose to use more 'forgiving' BED values and from this to calculate the re-irradiation dosefractionation schedule.

For protons, greater care is required since the inter-relationship between linear energy transfer (LET) and RBE can lead to significant over-dosage relative to accepted CNS tolerance doses, especially with the use of scanned proton beams. LET and RBE factors are important in order to deliver safe and effective retreatment doses. 


\section{Introduction}

The decision to recommend radiotherapy is never trivial, and is based on careful assessments of the potential benefits and associated risks. Re-treatment using photon-based Radiotherapy, although less commonly used, requires additional care in terms of patient selection, as either a radical or palliative approach to recurrent or metastatic tumour, or for new cancers occurring in an adjacent site. Parts of the central nervous system (CNS) are irradiated not only when treating tumours that originate in the CNS, but also when treating cancers that originate in other tissues. For example, thoracic treatments may incur a significant dose exposure to the spinal cord, and head and neck cancer therapy can involve a significant dose to areas of the CNS, such as the optic chiasm and brainstem, which have the same level of radiation tolerance as the spinal cord.

Over the past few years, a retreatment model has been developed that includes an allowance for the influence of the time between two separate photon-based radiation treatment courses $[1,2,3]$, along with many patient related safety features. The latest version of his model is now freely available as a downloadable Graphic User Interface (GUI) and is discussed in more detail below. It is specifically focused on spinal cord tolerance for photon-based treatments.

The increasing availability of proton therapy in many parts of the world raises the possibility of using the associated Bragg peak to reduce the normal tissue volumes irradiated in retreatment situations, especially where cancers arise in close proximity to previously irradiated tissues. However, as with photon retreatment, considerable care is required for patient selection with proton therapy and the technical aspects of treatment must be fully understood. Particularly important is the inter-relationship between linear energy transfer (LET), the 
relative biological effectiveness (RBE) and the degree of normal tissue dosesparing that can be achieved. This may be especially important when using scanned proton beams, since there is evidence that the RBE does not decline with tissue depth, when compared with the RBE reduction seen with passively scattered beams $[4,5,6]$. With scanned beams higher LET values can be found outside the clinical target volume, which is probably the result of a higher local fluence and hence shorter inter-track distances [7].

The present publication is specifically designed to initiate clinical users (clinicians and physicists) to the use of the GUI in a safe and relatively straight forward way. Users should attempt to replicate the results of the worked examples using the GUI. These examples are presented in a graduated way, from the relatively easy to the more difficult, ending with examples of proton retreatments. What needs to be done in order to make further progress towards safer and more effective re-treatments in other tumour sites is considered within the discussion below.

\section{Methods}

It is important to state that for any iso-effective calculations using either photon only treatments, or indeed for protons, with a photon-based numerator for RBE estimations, both need to be based on an iso-effective dose curve derived using the linear quadratic model using an $\alpha / \beta$ value of $2 \mathrm{~Gy}$, which is the most appropriate for CNS tissue. This value is used exclusively for all the BED estimates made throughout this publication. For a human standard tolerance dose equivalent to $50 \mathrm{~Gy}$ in 25 fractions of $2 \mathrm{~Gy}$, a $\mathrm{BED}_{\mathrm{TOL}}$ of $100 \mathrm{~Gy}_{2}$, is associated with a risk ( $\mathrm{R} \%)$ of $0.1185 \%)$, which is seen to be an excellent predictor of spinal tolerance to prevent the development of myelopathy (see Figure 1). The BED associated with a risk level of $0.1 \%\left(\mathrm{BED}_{0.1 \%}\right)$ is around 
99.32 $\mathrm{Gy}_{2}$ [3]. The principal data sets used for these estimates were mainly based on research by Kian Ang in humans (for the risk estimates) and in rhesus monkeys for the time course of recovery, and are given in detail in graphical form in an earlier publication [3]

\section{For photon therapy}

The spinal cord re-treatment model is described fully elsewhere [3] and readers are strongly advised to consult and be familiar with the concepts and features of the model. This model incorporates all the known data on spinal myelopathy following re-irradiation from animal experiments, which have been linked to:-

(1) a radiation-induced myelopathy dose-response curve for humans, adopting a degree of conservatism, which 'protectively' shifts the dose response curve slightly to the left,

(2) a dose-dependent recovery function which requires an initial operative threshold dose that is $35 \%$ of the BED for spinal cord tolerance. Beyond this threshold, recovery occurs more rapidly after a time interval of > 70 days.

Such links are necessary to comply with the results of the various experimental data sets.

The software rapidly estimates the BED of the given initial treatment course, expressed as a percentage of the designated tolerance BED. The time related function then provides an estimate of the BED that may be given to preserve a designated degree of risk if given in a designated number of fractions as a dose per fraction.

This is explained in detail for users of the downloadable graphical user interface (GUI). This is used to enter the initial total dose $\left(D_{\text {init }}\right)$ and number of 
fractions (n) used for that initial course of treatment, plus the level of risk considered acceptable $(\mathrm{R} \%)$ for a specific retreatment. These parameters are then automatically converted into the input parameter, $\mathrm{BED}_{1}(\%)$, which is the ratio of the $\mathrm{BED}$ initially delivered (termed $\mathrm{BED}_{\text {init }}$ ) divided by the $\mathrm{BED}$ associate with the treatment the risk considered acceptable at retreatment (termed $\mathrm{BED}_{\mathrm{R} \%}$ ).

Thus $\mathrm{BED}_{1}(\%)=\left(\mathrm{BED}_{\text {init }} / \mathrm{BED}_{\mathrm{R} \%}\right) \times 100$.

The degree of risk must be entered with care. As indicated the $\mathrm{BED}_{\mathrm{R}}$ value is based on the risk level accepted for re-treatment. In the GUI, the acceptable risk $\left(\mathrm{R}_{\%}\right)$ is set at a default value of $1 \%\left(\mathrm{BED}_{1 \%}=109.6 \mathrm{~Gy}_{2}\right)$, but this can be changed, for example to the more demanding level of $0.1 \%$ (one in a thousand risk, $\mathrm{BED}_{0.1 \%}-99.32 \mathrm{~Gy}_{2}$ ), or in specific circumstances increased. The risk level sets the threshold for the bio-effectiveness of initial and retreatment radiotherapy courses. The other input factor is the elapsed time $(t)$ between the initial and planned re-treatment, expressed in years.

It is then necessary to insert the number of dose fractions $\left(\mathrm{n}_{\mathrm{r}}\right)$ that might be ideally used for the re-treatment schedule. The model then estimates the output parameter $\mathrm{BED}_{2}(\%)$, which is the allowable re-treatment BED (referred to as $\left.\mathrm{BED}_{\text {ret }}\right)$, where $\mathrm{BED}_{2}(\%)=\left(\mathrm{BED}_{\text {ret }} /\right.$ acceptable $\left.\mathrm{BED}_{\mathrm{R} \%}\right) \times 100$. From this information, the GUI then automatically provides the dose per fraction for the proposed number of fractions for the re-treatment $\left(\mathrm{n}_{\mathrm{r}}\right)$. This could, for example, depend on whether the proposed purpose treatment is either curative or palliative. The acceptable BED used for retreatment calculations $\left(\mathrm{BED}_{\mathrm{R} \%}\right)$ is based on the risk acceptable on retreatment which is unlikely to be the maximum risk acceptable for the initial treatment, conventional tolerance $\left(\mathrm{BED}_{\mathrm{TOL}}\right)$. 
There is also a conservative factor $(\mathrm{C})$ range feature which essentially adjusts the original dose response curve to the left, thus reducing the threshold dose level of the bio-effect. The degree of conservatism required should be accessed by responsible clinicians in accordance with their assessment of the individual patient's clinical history, so that allowances can be made for previous surgery, chemotherapy, age, other medical conditions which can adversely affect tolerance, or combinations of these. Any value ranges between $0 \%$ to $20 \%$ conservatism (the later ultra-cautious) is permitted within the calculation programme. Mild chemotherapy, may suggest a 5\% change, but intensive chemotherapy with drugs that sensitise the CNS will demand more, say $10 \%$ and extremes of age should require at least 10\%. Significant de-vascularising surgery should require at least $10 \%$. Further discussion of this issue is available elsewhere [3].

The original Graphical User Interface (GUI) can be downloaded from the supplementary information provided with the original publication [3], with updates available at [http://thomaswoolley.co.uk/codes]. This facilitates estimates of allowable dose per fraction and number of fractions for retreatment. Estimates should be regarded as a boundary value not to be exceeded.

The GUI also includes a Graphic which shows the relationship between $\mathrm{BED}_{1}(\%)$ and $\mathrm{BED}_{2}(\%)$, with the additional option of the available rodent data being superimposed.

The responsible clinicians may then carefully review the recommended number of fractions and dose per fraction and decide whether any further reduction is necessary according to a particular clinical situation. A rounding down of the final estimated dose per fraction in the direction of safety is recommended. It is perhaps useful for clinicians to consider the analogy of the lines of a tennis court to represent the model results: these must be respected and the aim of the 
user should be to operate within those lines. The model essentially provides the maximum permissible dose per fraction which can always be rounded down or reduced to better comply with local protocols.

\section{For proton therapy}

There are a number of proton radiobiology models for the estimation of RBE [8]. In the present paper, the simple efficiency model for any ion beam is used for estimating the LET-determined proton RBE [9-12]. This incorporates saturation-effects for the relationship between the reference radiation radiosensitivities ( $\alpha$ and $\beta$ ) and their maximum values at the turnover positions of the LET/RBE relationships at a $\mathrm{LET}_{\mathrm{U}}$ value of $30.5 \mathrm{keV} / \mu \mathrm{m}$, which is compatible with the data of Belli et al [13]. A short Mathematica ${ }^{\circledR}$ (Wolfram Centre, Long Hanborough, Oxfordshire, UK) code for estimating the RBE of protons for different LET value and dose/fraction, and based on the reference radiation (photon) $\alpha$ and $\beta$ radio-sensitivities, is given in the Appendix (B). For the CNS tissue $\alpha=0.06 \mathrm{~Gy}^{-1}$ and $\beta=0.03 \mathrm{~Gy}^{-2}$ values were assumed to provide $\mathrm{a} / \beta$ ratio of $2 \mathrm{~Gy}$ within the RBE estimates. These RBE values can then be used to convert proton dose to photon dose or vice versa. All doses used refer to the physical dose: the Gy-RBE dose convention for protons is not used in order to avoid confusion. Graphical displays of the biological effective dose (BED) are estimated for protons, assuming variable LET values of 2, 3, 5, 7.5 and 10 $\mathrm{keV} / \mu \mathrm{m}$, which covers the range found by Grassburger et al in their clinical studies [14], but assumes a reference LET of around $0.22 \mathrm{keV} / \mu \mathrm{m}$. For the conventional international policy of adopting a single RBE of 1.1 for proton therapy, regardless of dose per fraction and LET, then the reference radiation $\mathrm{BED}$ is assumed to be identical to the proton treatment BED for comparative purposes. 
The degree of normal tissue dose sparing is defined as the dose in the critical organ at risk divided by the prescribed tumour dose and expressed as a percentage.

In the case of treatments with protons, either for re-treatment or for each of two treatment courses, the GUI can still be used, but it is essential to use a photon equivalent dose/fraction input (see Appendix (A)), as well as to convert the final estimated photon dose/fraction, into an appropriate proton dose, using the estimated RBE.

The GUI [3] was used to generate the results given below in the form of a number of worked examples.

\section{Results}

An iso-effect curve, utilising a 2 Gy $\alpha / \beta$ value for CNS tissue [15], is shown in Figure 1, along with points that represent a variety clinically varied 'safe' dosefractionation schedules and more typically prescription doses for the treatment of cancer. This data forms the basis of the calculations presented below, including the numerator for any proton RBE estimates. 


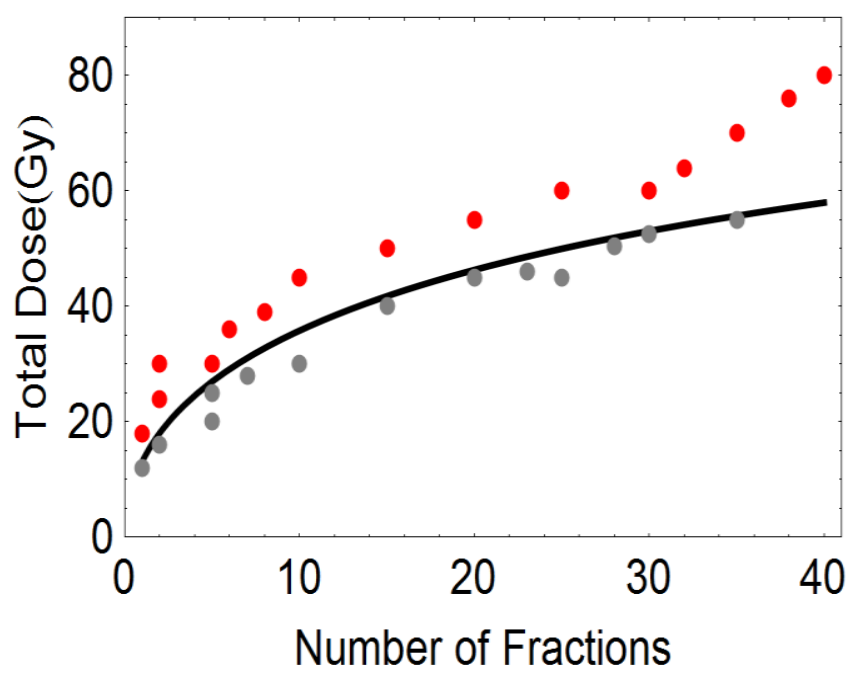

Figure 1: Total Dose is plotted against number of dose fractions yielding the curve shown for spinal cord paralysis, obtained using the LQ model with $\alpha / \beta=2$ Gy for an iso-effect to the standard tolerance value of $50 \mathrm{~Gy}$ in 25 fractions of $2 \mathrm{~Gy}$. The grey points correspond to 'safe' treatment schedules previously used by the first author. The red points refer to dose fractionation schedules often used to treat squamous cell cancers, which exceed the predictions of the iso-effect curve. Clinical radiotherapy may utilise the grey dose-fractionation data points for palliative cancer treatment close to the spinal cord. The red data points represent situations where curative tumour doses are given. Great care is taken to ensure to obtain sufficient spinal cord dose sparing so that the spinal cord total doses are below the curve [reproduced with permission of Institute of Physics Publishing from Jones (2017) [15].

\section{Worked Examples}

In working through the following worked examples, which are related to either photon (examples 1-4) or proton (examples 5-6) retreatments, it is very strongly recommended that the text is read in conjunction with direct reference to the GUI, as provided in Wooley et al [3], since this will greatly enhance the understanding of what data was entered, in order to undertake the calculations, and to how the results are illustrated. For this reason the terminology and abbreviations used in the present text directly match those displayed on the GUI, In practice the GUI only accepts the basic input information of previous total dose, number of fractions and demands entry of the number of retreatment 
dose fractions. The essential further input is the risk level, and since this is set at a default value of $1 \%$ might require to be changed to lower or higher risk levels depending on the clinical situation.

\section{Photon treatments}

Worked Example 1. After a maximum initial spinal cord dose of 46.5 Gy in 30 fractions, to a fit young patient, it is decided to re-treat that patient 18 months later. There is a history of two separate intervening 6 cycle courses of chemotherapy, containing Cis-platinum and anthracycline, but no history of any spinal surgery. What maximum dose could be given at retreatment in either10, 15 , or 20 fractions if a conservative factor of around $10 \%$ is used?

The resulting proposed retreatment doses/fraction for the differing proposed fraction numbers are given in Table 1, with suggested downwards adjustment of the estimated doses provided by the GUI to deliver a practical safe total dose. The GUI derived $\mathrm{BED}_{0.1 \%}$ used in the calculations; including conservatism $(10 \%)$ was $88.36 \mathrm{~Gy}_{2}$.

Table 1. Estimated treatment parameters obtained using the GUI for worked example 1 for a $0.1 \%$ risk of myelopathy after retreatment.

\begin{tabular}{|l|l|l|l|}
\hline GUI input settings: Risk $=0.1 \%$, conservative factor $=10.0 \%, \mathrm{t}=1.5 \mathrm{yr}$ \\
\hline $\begin{array}{l}\text { Number of dose } \\
\text { fractions }\left(\mathrm{n}_{\mathrm{r}}\right)\end{array}$ & 10 & 15 & 20 \\
\hline $\begin{array}{l}\text { Estimated } \\
\text { dose/fraction }(\mathrm{Gy})\end{array}$ & 2.97 & 2.3 & 1.9 \\
\hline $\begin{array}{l}\text { Practical } \\
\text { dose/fraction }(\mathrm{Gy})\end{array}$ & 2.9 or 2.8 & 2.25 or 2.2 & 1.85 or 1.8 \\
\hline
\end{tabular}


Worked Example 2. After an initial palliative treatment, prescribed as $20 \mathrm{~Gy}$ in 5 fractions, if re-treatment proves necessary. There are no adverse factors likely to influence CNS tolerance. The maximum initial dose to a section of the spinal cord was 20.8 Gy (4.16 Gy/fraction). What re-treatment dose can be permitted for time intervals of 3, 6 and 12 months prior to re-treatment, given either as a single does or as 5 or 10 dose fractions?

The prosed retreatment single doses and doses/fraction for the differing fraction numbers are given in Table 2, which also includes suggested practical doses, in parentheses, after the estimated doses.

Table 2. Estimated treatment parameter using the GUI for worked example 2 for a $0.1 \%$ risk of myelopathy after retreatment.

\begin{tabular}{|c|c|c|c|}
\hline \\
\hline \multicolumn{4}{|c|}{ 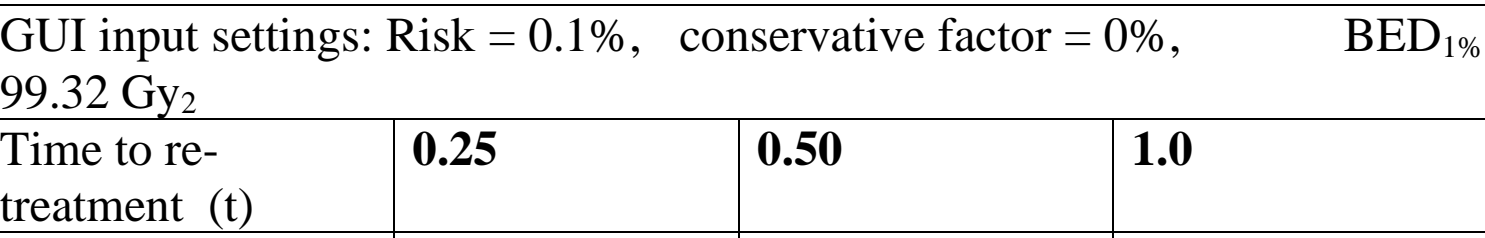 } \\
\hline \multicolumn{4}{|c|}{\begin{tabular}{l|l|l|l} 
& 12.02 \\
Single dose $)$ & $(8)$ & $(10)$ & $(10-11)$
\end{tabular}} \\
\hline $\begin{array}{l}\text { Re-treatment } \\
\text { dose/fraction (Gy) } \\
\text { (5 fractions) }\end{array}$ & $\begin{array}{l}3.51 \\
(3.4)\end{array}$ & $\begin{array}{l}4.43 \\
(4)\end{array}$ & $\begin{array}{l}4.89 \\
\text { (4 to } 4.5 \text { ) }\end{array}$ \\
\hline $\begin{array}{l}\text { Re-treatment } \\
\text { dose/fraction (Gy) } \\
\text { (10 fractions) }\end{array}$ & $\begin{array}{l}2.26 \\
(2.2)\end{array}$ & $\begin{array}{l}2.9 \\
(2.8)\end{array}$ & $\begin{array}{l}3.23 \\
(3)\end{array}$ \\
\hline
\end{tabular}

Special care is required for clinical situations that occur within 1-6 months, especially if the 'initial treatment course' has a low BED value relative to $\mathrm{BED}_{\mathrm{R} \%}$, as in the following practical examples. 
Worked Example 3. After separate but consecutive treatments for 2 thoracic spinal column metastases (vertebrae, D8 and D10), using a rapidly delivered Linac based treatment technique in a single treatment session, a new metastasis arises in D9, 3 months later, and further treatment is given one month after that, i.e. 4 months after the initial dose. There are no adverse factors likely to influence CNS tolerance. The cumulative spinal cord dose near to D9 from the initial treatments was estimated to be $3 \mathrm{~Gy}$. What dose can now be given there as a rapidly delivered single dose or in three fractions, assuming either a $0.1 \%$ or a $1 \%$ level of risk?

Note that the $\mathrm{BED}_{\text {init }}$ will be reduced by the duration of time taken to deliver the treatment of the two initial lesions, but and since the treatment is given 'rapidly' the influence of repair during treatment [16] is considered to be neglected in order to provide a safer constraint. This given dose cannot be ignored in any subsequent treatment. For a single 3 Gy treatment, the maximum $\mathrm{BED}_{\text {init }}$ is $3(1+3 / 2)=7.5 \mathrm{~Gy}_{2}$. Based on this potential overestimate of the initial BED, the estimated re-irradiation doses are given in Table 3, for either a $1 \%$ or $0.1 \%$ level of acceptable risk for a single treatment session and for 3 separate treatment sessions. The results are given in Table 3 . The GUI derived $\mathrm{BED}_{1 \%}$ and $\mathrm{BED}_{0.1 \%}$ values used in calculations were $109.6 \mathrm{~Gy}_{2}$ and $99.32 \mathrm{~Gy}_{2}$, respectively.

Table 3. Estimated maximum treatment dose obtained using the GUI for worked example 3, for different risks of myelopathy on retreatment, with suggested practical doses given in parentheses.

\begin{tabular}{|l|l|l|}
\hline \multicolumn{3}{|c|}{ GUI initial settings: conservative factor $=0 \%, \mathrm{t}=0.32$ years } \\
\hline & Risk $=1 \%$ & Risk $=0.1 \%$ \\
\hline 1 treatment session & $13.32 \mathrm{~Gy}(13 \mathrm{~Gy})$ & $12.59 \mathrm{~Gy} \quad(12 \mathrm{~Gy})$ \\
\hline 3 treatment sessions & $7.31 \mathrm{~Gy}(7 \mathrm{~Gy})$ & $6.89 \mathrm{~Gy} \quad(6.5 \mathrm{~Gy})$ \\
\hline
\end{tabular}

The next example considers a long delay occurring early within an interrupted treatment course. 
Worked Example 4. A young, fit patient received 10.8 Gy in 6 fractions as part of an intended full course treatment of 45 Gy in 25 fractions $\left(B E D_{\text {init }} 85.5\right.$ $\left.G y_{2}\right)$, but they defaulted from therapy for 5 weeks. What subsequent dose might then be given if the acceptable risk were taken to be either $1 \%$ or $0.01 \%$ ? There are no adverse factors likely to influence CNS tolerance.

On re-starting treatment, the previously delivered dose cannot be ignored. Spinal tolerance would be exceeded if the previously dose, given in 6 fractions, was neglected and the previously intended full treatment dose given as a second course, since the total BED would then be $10.8(1+1.8 / 2)+45(1+1.8 / 2)=106.02$ $\mathrm{Gy}_{2}$, which exceeds conventional tolerance $\left(\mathrm{BED}_{\mathrm{TOL}}=100 \mathrm{~Gy}_{2}\right)$ and could even be exceeded in regions with doses which exceed the prescribed dose. The initial irradiation was associated with a $\mathrm{BED}_{\text {init }}$ of $20.52 \mathrm{~Gy}_{2}$. The results are presented in Table 4, with a slightly higher dose/fraction for the remaining 19 fractions, or at a reduced dose/fraction for a revised 24 fraction schedule. The GUI derived $\mathrm{BED}_{1 \%}$ and $\mathrm{BED}_{0.1 \%}$ values used in calculations were $109.6 \mathrm{~Gy}_{2}$ and $99.32 \mathrm{~Gy}_{2}$, respectively. No allowance for tumour repopulation has been made; this would require separate estimations of the tumour BED depending on the tumour type [17-18].

Table 4. Estimated results using the GUI for worked example 4, with suggested practical doses suggested in parentheses

\begin{tabular}{|l|l|l|}
\hline \multicolumn{3}{|c|}{ GUI initial settings: conservative factor $=0 \%, \mathrm{t}=0.1$ years } \\
\hline & Risk $=1 \%$ & Risk $=0.1 \%$ \\
\hline 19 Fractions & $2.22 \mathrm{~Gy}(2.1 \mathrm{~Gy})$ & $2.05 \mathrm{~Gy} \quad(2 \mathrm{~Gy})$ \\
\hline 24 Fractions & $1.90 \mathrm{~Gy}(1.8 \mathrm{~Gy})$ & $1.75 \mathrm{~Gy} \quad(1.7 \mathrm{~Gy})$ \\
\hline
\end{tabular}




\section{Proton Treatments}

A plot showing the relationship between the degree of normal tissue dose sparing and the BED for protons with differing LET values is given in Figure 2. The normal tissue sparing is defined as the ratio of dose to the optic chiasm divided by the prescribed tumour dose and is expressed as a percentage. Three horizontal lines are also displayed Figure 2, indicative of the BED values for the standard neurological tolerance and also for a 10 or $20 \%$ level of conservatism (i.e. BED values of 100,90 or $80 \mathrm{~Gy}$, respectively), which would refer to equivalent photon doses in 2 Gy fractions of 50 Gy, $44-46$ Gy and 40 Gy. The lowermost curve is that of the reference radiation (megavoltage photons) but also applies for protons, but only on the assumption that a fixed RBE of 1.1 is correct, and so exactly matches the BED of the reference radiation. If this assumption is incorrect [19], then the LET-RBE modelled BED values, which vary with LET, are probably more realistic. The information displayed in Figure 2 is based on the example of a prescribed total tumour dose of $70 \mathrm{~Gy}$, given in 35 fractions, for an ethmoid sinus cancer described in a publication by Grassburger et al [14], where the LET at the level of the optic chiasm was around $7.5 \mathrm{keV} / \mu \mathrm{m}$, and to have received a dose of $24.5 \mathrm{~Gy}$, that is around $35 \%$ of that prescribed to the tumour (i.e. the degree of normal tissue sparing is $35 \%$ ). This appears to be safe, because the three BED tolerance levels are not breached (i.e. the optic chiasm has received lower BED values below tolerance) if the dose-sparing is below approximately $40 \%$.

It can be seen that the degree of normal tissue dose sparing, relative to the prescribed dose, must be increased to counteract the effect of increasing LET, and in any re-treatments it is recommended that the LET determined BED values are used in calculations if protons are used after initial photon treatment or in the case of two proton courses, as in the following worked examples. 


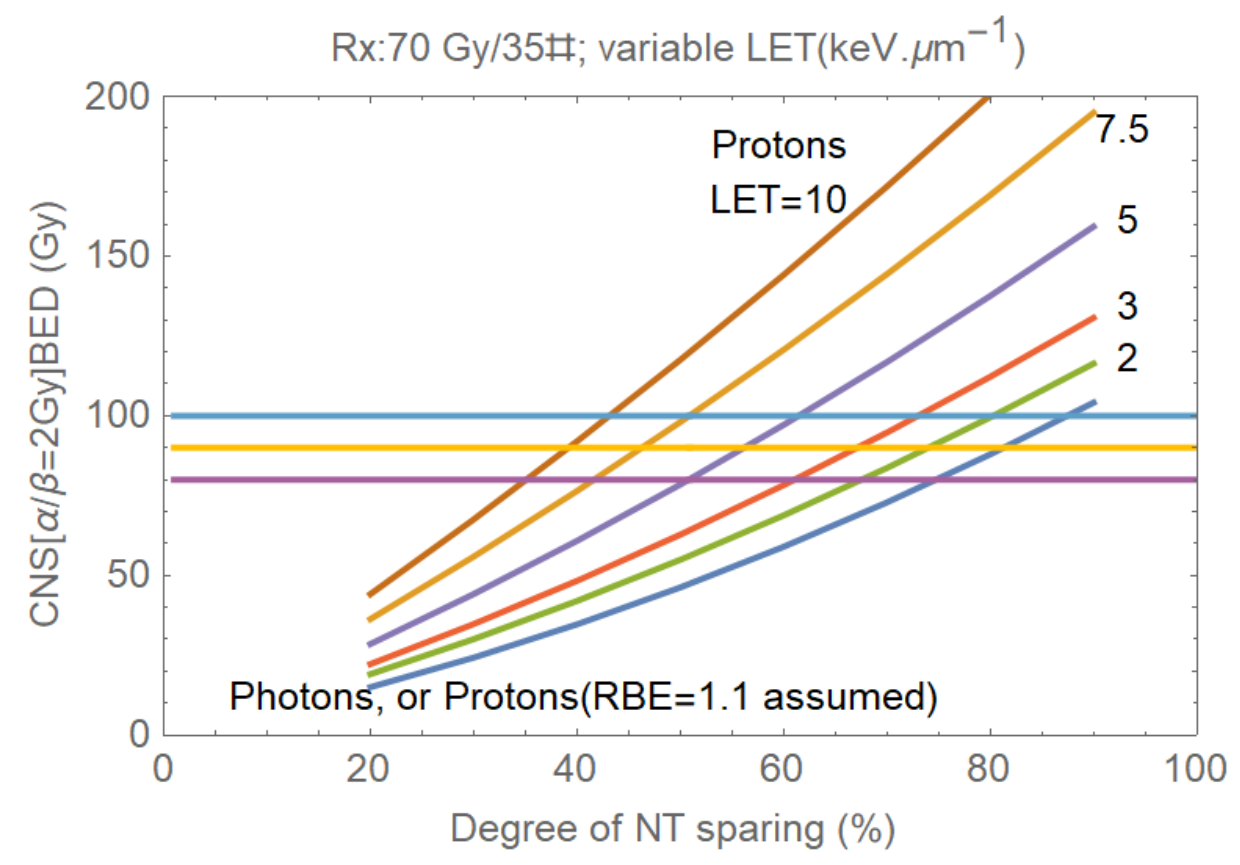

Figure 2: Plots of CNS BED (using an $\alpha / \beta=2$ Gy) against the percentage degree of achievable normal tissue dose sparing for variations in proton LET values between 2 and 10 $\mathrm{keV} / \mu \mathrm{m}$. The lower most curve plotted is that for a reference megavoltage photon radiation, which would be identical to that for a proton treatment had a fixed RBE of 1.1 been assumed to produce an iso-effect. The horizontal lines represent three possible CNS tolerance levels for spinal cord and other serially organised nervous tissues such as the brainstem and optic chiasm, which from above downwards are namely BED values of 100, 90 and $80 \mathrm{~Gy}_{2}$ which respectively represent $0 \%$ conservatism (blue), 10\% conservatism (yellow) and 20\% conservatism (purple). The higher LET values shown have been linked to a RBE model that produces higher BEDs values for the CNS. For patient safety, the treatment conditions should fall below the relevant horizontal lines, which require a more favourable degree of normal tissue sparing.

Worked Example 5. An initial radiation treatment is associated with a spinal cord dose of 47.5 Gy in 30 fractions of photons (1.58 Gy/fraction). For the treatment of a recurrence, 18 months later, proton therapy is proposed. Treatment planning provides two choices for a proton physical dose per fraction of 1.6 Gy, associated with either a spinal cord LET of 1.5 or $5 \mathrm{keV} / \mu \mathrm{m}$. There is no other history that might influence spinal cord tolerance. What dose could be given if a $0.1 \%$ risk of myelopathy is accepted? Compare the results with those based on the use of a fixed RBE of 1.1.

(a) For re-treatment using a LET of $1.5 \mathrm{keV} / \mu \mathrm{m}$, the RBE for the spinal cord $(\alpha / \beta=2$ Gy) is estimated to be 1.14 (see Appendix B for method). Thus based 
on retreatment given in 23 fraction the GUI indicates an estimated total retreatment dose of $43.01 \mathrm{~Gy}$ given at $1.87 \mathrm{~Gy} /$ fraction (photons), or a $\mathrm{BED}_{\text {ret }}$ of $1.87 \times 23(1+1.87 / 2)=83.23 \mathrm{~Gy}_{2}$. The proposed proton dose/fraction of $1.6 \mathrm{~Gy}$ would, based on the estimated RBE of 1.14 , be equivalent to $1.82 \mathrm{~Gy} /$ fraction of photons $(1.6 \mathrm{~Gy} \times 1.14)$. This is acceptably close to, but lower than, 1.87 Gy/fraction calculated using the GUI and is consequently safer. The proposed re-treatment dose using protons is thus 23 fractions of $1.6 \mathrm{~Gy}$.

(b) For re-treatment using an LET of $5 \mathrm{keV} / \mu \mathrm{m}$, the spinal cord RBE is now estimated to be 1.47 for again a proton dose/fraction of $1.6 \mathrm{~Gy}$ (see Appendix B). From the calculation in section (a) the GUI indicates a re-irradiation photon $\mathrm{BED}_{\text {ret }}$ of $83.23 \mathrm{~Gy}_{2}$. Since the photon equivalent dose of this higher LET beam for a $1.6 \mathrm{~Gy} /$ fraction re-treatment is now higher, namely $2.35 \mathrm{~Gy}(1.47 \times 1.6 \mathrm{~Gy})$ of photons, a reduced number of dose fractions must be used if the planned dose/fraction is to be retained. This is found by solving for $\mathrm{N}$ in the equation $\mathrm{N} \times 2.35(1+2.35 / 2)=83 \mathrm{~Gy}_{2}$ (the $\mathrm{BED}_{\text {ret }}$ being slightly rounded down from 83.23 to $83 \mathrm{~Gy}_{2}$ ). The calculated value of $\mathrm{N}(16.2)$ is then rounded down; the use of a dose of 16 fractions of 1.6 Gy is proposed for these higher LET protons.

(c) For a generic fixed RBE of 1.1, the retreatment dose/fraction equivalent of $1.6 \mathrm{~Gy}$ of protons would be $1.76 \mathrm{~Gy}$ of photons. In order to achieve a $\mathrm{BED}_{\text {ret }}$ value of around $83 \mathrm{~Gy}_{2}$, would require a total of 25 fractions [found by solving the equation $\mathrm{N} \times 1.76(1+1.76 / 2)=83 ; \mathrm{N}=25$ ], rather than 23 fractions for a more appropriate RBE of 1.14, and 16 fractions for a higher RBE of 1.47 .

For each case the actually delivered $\mathrm{BED}_{\text {ret }}$ for 25 fractions of $1.6 \mathrm{~Gy}$, initially based on a generic RBE of 1.1 can then be estimated:-

(a) $25 \times 1.6 \times 1.14(1+1.6 \times 1.14 / 2)=87.19 \mathrm{~Gy}_{2}$ for $\mathrm{RBE}=1.14$ and 
(b) $25 \times 1.6 \times 1.47(1+1.6 \times 1.47 / 2)=127.95 \mathrm{~Gy}_{2}$ for the RBE of 1.47 .

These values are both significantly above the $\mathrm{BED}_{\text {ret }}$ value of $83.23 \mathrm{~Gy}_{2}$ calculated by the GUI and so would be associated with a significantly increased risk of myelopathy, namely $0.25 \%$ for an LET of $1.5 \mathrm{keV} / \mu \mathrm{m}$ and $55 \%$ for an LET of $5 \mathrm{keV} / \mu \mathrm{m}$. Even for an initial dose, not involving retreatment, the respective equivalent total doses in 2 Gy fractions (EQD-2) would have been 43.6 Gy and $63.97 \mathrm{~Gy}$, with only the former being safe.

Worked Example 6: A Proton therapy treatment is given, such that the optic chiasm received 30 fractions of $1.3 \mathrm{~Gy}$ (physical dose). Two years later, with no adverse history, re-treatment is required. What retreatment dose would be safe for a $0.1 \%$ risk under the following conditions:-

(a) A proton LET of $3 \mathrm{keV} / \mu \mathrm{m}, \mathrm{RBE}$ of 1.32 , an equivalent photon dose of $1.716 \mathrm{~Gy} /$ fraction or a total equivalent photon dose of $51.48 \mathrm{~Gy}$; a $\mathrm{BED}_{\text {int }}$ of $95.65 \mathrm{~Gy}_{2}$,

(b) A proton LET of $1.5 \mathrm{keV} / \mu \mathrm{m}, \mathrm{RBE}$ of 1.15 , an equivalent photon dose $1.495 \mathrm{~Gy} /$ fraction or a total with equivalent total dose of $44.85 \mathrm{~Gy}$; a $\mathrm{BED}_{\text {int }}$ of $78.38 \mathrm{~Gy}_{2}$.

If retreatment is also given in 30 fractions and with the same assumed LET values, and by using the above equivalent total doses in 30 fractions the GUI estimates (a) $1.57 \mathrm{~Gy}$ and (b) $1.67 \mathrm{~Gy} /$ fraction of photons, which respectively provide $\mathrm{BED}_{\text {ret }}$ values of 83.7 and $91.62 \mathrm{~Gy}_{2}$ for re-treatment.

It is then necessary to estimate the number of $1.3 \mathrm{~Gy}$ fractions of proton to provide these photon $\mathrm{BED}_{\text {ret }}$ values. 
For (a) this is found by solving for $\mathrm{N}$ the following equation:

$\mathrm{N} \times 1.3 \times 1.32(1+1.3 \times 1.32 / 2)=83.7$; or $\mathrm{N}=26.25$, i.e. 26 fractions of $1.3 \mathrm{~Gy}$ for protons.

For (b) this is found by solving for $\mathrm{N}$ in the following equation:

$\mathrm{N} \times 1.3 \times 1.15(1+1.3 \times 1.15 / 2)=91.62 ; \mathrm{N}=35.07$, i.e 34 fractions of $1.3 \mathrm{~Gy}$ for protons.

\section{Discussion}

The clinical literature on the issue of re-treatment is complex, consisting largely of retrospective data, often without reference to BED values determination or the associated EQD2 derivation and with insufficient attention given to the role of the elapsed time between the initial and re-treatment courses, a continuous variable, which must reflect tissue recovery.

Clinicians must be extremely cautious in the interpretation of any currently published data, which may claim that a specified total re-treatment dose can be given, without using a BED or EQD-2 approach to correct for fractionation effects, unless such conclusions are based only on low dose/fraction radiotherapy in both the initial and subsequent re-treatment courses. Also, the notion that re-treatment is possible after a single time point has elapsed, e.g. 6 months or a year following the initial treatment course, is unfounded. This does not give sufficient flexibility, since time is a continuous variable and the safe dose will increase with time after any initial treatment and will also depend on the size of that initial dose.

It is also important for clinicians to specify the most relevant iso-dose surface, or tissue positions, at which any re-treatment calculations should be made. This applies to all forms of radiotherapy and especially where significant dose 
gradients are present, as in radio-surgical applications or if protons are used (where in addition LET maps may be especially informative).

The re-treatment model used is dependent on the validity of the linear quadratic (LQ) model, which appears to fit longstanding data based on clinical experience well, as shown in Figure 1. It is important to realise that the LQ model is itself conservative and protective for normal tissues with low $\alpha / \beta$ values: if straightening of the dose response curve does sometimes occur at higher doses (e.g. between say 7-12 Gy in some in vitro experiments, although rarely is the associated increase in treatment time for the large doses considered when doses are delivered at a fixed dose-rate), then the LQ predicts lower survival fractions, and lower iso-effective doses. Further descriptions of how to model such effects is provided by Jones and Dale [2019] and are at present only considered appropriate only for BED estimates in tumour.

It remains to be seen if more experiments in animals will be performed to establish further knowledge, for example on the effects of relatively low dose exposure in the initial course of treatment, and their influence at subsequent retreatment times? Experiments in primates are costly and there are ethical considerations making further work difficult unless precise clinical questions need to be asked that can only be addressed adequately in primates. Alternative approaches could include the setting up of a national or international data bases, allowing an analysis of similar groups of patients and studies/protocols that could also allow more precise analysis of the allowances that should be made for chemotherapy effects, local surgery/pressure effects/trauma, age, medical conditions etc [20].

Acknowledgements: The authors are especially grateful to colleagues Eamonn Gaffney and Thomas Woolley in the Department of Mathematics at Oxford and Cardiff, respectively. 
Declarations: This work was not grant supported, however, the authors are grateful for a travel grants to enable them to present this work in Stockholm, September 2018 as part of the Retreatment Symposium

\section{References:}

1. Jones B. Grant W. Retreatment of central nervous system tumours. Clinl Oncol 2014;6: 407-18.

2. Jones B Hopewell JW. Alternative models for estimating the radiotherapy retreatment dose for the spinal cord. Int J Radiat Biol 2014;90:731-41.

3. Woolley TE, Belmonte-Beitia J, Calvo GF, Hopewell JW, Gaffney EA, Jones B. Changes in the retreatment radiation tolerance of the spinal cord with time after the initial treatment. Int J Radiat Biol 2018;94:515-31.

4. Calugaru V, Nauraye C, Noel G, Giocanti N, Favaudon V, Megnin-Chanet F. Radiobiological characterization of two therapeutic proton beams with different initial energy spectra used at the Institut Curie Proton Therapy Center in Orsay. Int J Radiat Oncol, Biol, Phys 2011;81:1136-43.

5. Britten RA, Nazaryan V, Davis LK, Klein SB, Nichiporov D, Mendonca MS, Wolanski M, Nie X, George J. Keppel C. Variations in the RBE for cell killing along the depth-dose profile of a modulated proton therapy beam. Rad Res 2013;179:21-8.

6. Jones B, McMahon SJ, Prise KM. The Radiobiology of Proton Therapy: Challenges and opportunities around relative biological effectiveness. Clin Oncol 2018; 30:285-92.

7. Jones B. Chapter 1 in 'Practical Radiobiology for Proton Therapy planning', Institute of Physics Publishing (Bristol \& Philadelphia), 2017. ISBN 978-0-7503-1338-4 or 978-0-75031339-1.

8. Rørvik E, Fjæra LF, Dahle TJ, Dale E, Engeseth GM, Stokkevåg CH, Thörnqvist S, YtreHauge KS. Exploration and application of phenomenological RBE models for proton therapy. Phys Med Biol 2018 63:185013. https://doi.org/10.1088/1361-6560/aad9db

9. Jones B. Towards achieving the full clinical potential of proton therapy by inclusion of LET and RBE Models. Cancers 2015;7:460-80.

10. Jones B. A simpler energy transfer efficiency model to predict relative biological effect (RBE) for Protons and Heavier Ions. Front Oncol 2015;5:184. https://doi:10.3389/fonc.2015.00184 Erratum Front Oncol. 2016;6:32. https://doi:10.3389/fonc.2016.00032

11. Jones B Clinical radiobiology of proton therapy: modeling of RBE. Acta Oncol 2017;56:1374-378.

12. Jones B. Chapters $8 \& 9$ in 'Practical Radiobiology for Proton Therapy planning', Institute of Physics Publishing (Bristol \& Philadelphia), 2017. ISBN 978-0-7503-1338-4 or 978-07503-1339-1.

13. Belli M, Bettega D, Calzolari P, Cera F, Cherubini R, Dalla Vecchia M, Durante M, Favaretto S, Gialanella G, Grossi G. Inactivation of human normal and tumour cells irradiated with low energy protons. Int J Radiat Biol 2000;76:831-9.

14. Grassberger C, Trofimov A, Lomax A, Paganetti H. Variations in linear energy transfer within clinical proton therapy fields and the potential for biological treatment planning. Int J Radiat Oncol. Biol, Phys 2011;80:1559-66. 
15. Jones B. Chapters 2 in 'Practical Radiobiology for Proton Therapy planning', Institute of Physics Publishing (Bristol \& Philadelphia), 2017. ISBN 978-0-7503-1338-4 or 978-0-75031339-1.

16. Jones B. Hopewell JW. Modelling the influence of treatment time on the biological effectiveness of single radiosurgery treatments: Derivation of 'protective' dose modification factors. Brit J Radiol 2018;92:20180111 https://doi.org/10.1259/bjr.20180111

17. Dale RG, Hendry JH, Jones B, Deehan C, Sinclair JA. Practical methods for compensating for missed treatment days in radiotherapy, with particular reference to head \& neck schedules. Clin Oncol 2002;14:382-93.

18. Jones B. Dale RG. The evolution of practical radiobiological modelling, Brit J Radiol 2019; 92: 20180097. https://doi.org/10.1259/bjr.20180097

19. Jones B. Why RBE must be a variable and not a constant in proton therapy. Brit J Radiol. 2016;89:20160116. http://dx.doi.org/10.1259/bjr.20160116

20. R. Muirhead, B. Jones. Re-irradiation is now a real option - But how do we take it forward? Clin Oncol 2018;30:65-66

21. Jones B and Dale RG. The evolution of practical radiobiological modelling. Brit J Radiol. 92 (1093) 20180097 https://doi.org/10.1259/bjr.20180097 


\section{Appendix}

A: The photon equivalent doses $\left(\mathrm{d}_{\mathrm{eq}}\right)$ are the physical proton dose $\times \mathrm{RBE}$, and can be used within the standard BED equation, namely:

$$
\mathrm{BED}=\mathrm{N} \cdot \mathrm{d}_{\mathrm{eq}}\left(1+\mathrm{d}_{\mathrm{eq}} / 2\right) \text {, where } 2 \text { Gy is the } \alpha / \beta \text { ratio. }
$$

B: To obtain RBE values

(1) Programming the Simple efficiency model LET-RBE in Mathematica ${ }^{\circledR}$ [http://www.wolfram.com/mathematica/] uses the following definitions and steps. References 9-12 provide further information.

The control megavoltage photon reference LET is given by $l e t_{c}=0.22 \mathrm{keV} / \mu \mathrm{m}$

The maximum bio-efficiency LET for protons, let $_{u}$, is assumed to be $30.5 \mathrm{keV} / \mu \mathrm{m}$, the proton physical dose is allocated as $d_{h i}$. Further values are allocated for:

the reference-radiation $\beta$ value; $\beta$ low, the reference-radiation $\alpha$ value; $\alpha$ low, the $\alpha / \beta$ ratio is then expressed as $\alpha$ low/ $\beta$ low;

The operative LET value is $l e t_{x}$ and a value is allocated e.g. let $_{x}=5$;

The $\alpha$ value at the maximum bio-effectiveness $(\alpha u)$ is

$$
\alpha u=10.57 / 3.92(1-\operatorname{Exp}[-3.92 \alpha \text { low }]) ;
$$

The $\alpha$ value at the operative LET is then given by

$$
A h i=\alpha l o w+(a u-\alpha l o w)\left(\text { let }_{x}-\text { let }_{c}\right) /\left(\text { let }_{u}-\text { let }_{c}\right) ;
$$

Likewise for $\beta$, the value at the maximum bio-effectiveness is

$$
B u=0.06(1-\operatorname{Exp}[-50 \beta l o w]) ;
$$

The $\beta$ value at the operative LET is then

$$
B h i=\beta l o w+(\beta u-\beta \text { low })\left(\text { let }_{x}-\text { let }_{c}\right) /(\text { letu-letc }) ;
$$

The $\mathrm{d}_{\text {low }}$ dose is then found to be:

Dlow $=1 /(2$ blow $) \cdot\left(-\right.$ alow + Sqrt $\left[a l o w^{2}+4\right.$ ahi blow dhi +4 bhi blow dhi $\left.{ }^{2}\right]$;

This is divided by the $d h i$ proton dose to give the RBE (rbe), namely

$$
\text { Rbe }=\text { dlow/dhi; }
$$


(2) The resulting short Mathematica ${ }^{\circledR}$ [http://www.wolfram.com/ mathematica/] code will provide an estimate of RBE after entering values for dhi and letx:

$\mathrm{dhi}=\ldots$; letx $=\_$;

blow=alow/2; alow=0.06; au=10.57/3.92 (1-Exp[-3.92 alow]);

ahi=alow+(au-alow) (letx-letc)/(letu-letc);

bu $=0.06$ (1-Exp[-50 blow]);

bhi=blow+(bu-blow) (letx-letc)/(letu-letc);

dlow $=1 /(2$ blow $)\left(-\right.$ alow $+\backslash[$ Sqrt $]\left(\right.$ alow $^{2}+4$ ahi blow dhi+4 bhi blow dhi $\left.\left.^{2}\right)\right)$;

rbe=dlow $/$ dhi;

letc $=0.22$; letu $=30.5$; print rbe 